\title{
The Sre Substrate Tks5, Podosomes (Invadopodia), and Cancer Cell Invasion
}

\author{
S.A. Courtneidge, ${ }^{* \dagger}$ E.F. Azucena JR. ${ }^{* \dagger}$ I. Pass, ${ }^{* \dagger}$ D.F. Seals, ${ }^{*}$ and L. Tesfay* \\ "The Van Andel Research Institute, Grand Rapids, Michigan 49505; ${ }^{\dagger}$ The Burnham Institute, \\ La Jolla, California 92037
}

\begin{abstract}
Some years ago, we employed a screen of phage cDNA expression libraries to identify novel substrates of the protein tyrosine kinase Src. One of these, Tks5 (previously known as Fish), is a large scaffolding protein with an amino-terminal PX domain and five SH3 domains. In normal fibroblasts, Tks5 is cytoplasmic, but the protein is found in podosomes when the cells are transformed with Src. Using short interfering RNA technology, we have shown that Tks5 is required for podosome formation. Furthermore, cells with reduced Tks5 expression are poorly invasive through Matrigel. Tks5 is expressed and localized to podosomes in invasive human cancer cell lines and in tumor tissue, particularly breast cancers and melanomas. In these cells too, Tks5 is required for invasion. Our future work will focus on the identification of the binding partners of Tks5 that are responsible for podosome formation and invasion, and on determining the role of Tks5 in animal models of metastasis.
\end{abstract}

The study of tumor viruses has contributed much to our understanding of the mechanisms by which cancer cells arise, resist signals that restrain the growth of normal cells, and metastasize. The study of the oncogene $s r c$ has been particularly informative. Src-transformed cells are morphologically transformed and highly invasive both in vitro and in vivo. The $s r c$ gene product is a membrane-associated protein tyrosine kinase. Since the intrinsic catalytic activity of Src is absolutely required for transformation, identifying and studying its substrates has proved invaluable in understanding many aspects of the cancer phenotype, including factor-independent growth, motility, and escape from apoptosis (Martin 2001; Frame 2002).

Several years ago, we developed a screen to rapidly isolate Src substrates, regardless of their abundance or ability to associate with Src (Lock et al. 1998). This involved using an enriched preparation of Src to phosphorylate proteins produced from phage expressing mammalian cDNA libraries. Positive clones were identified by immunoblotting with anti-phosphotyrosine antibodies. Our screen was validated by the cloning of many established Src substrates. We also implicated several known proteins as Src substrates, and we cloned several novel cDNAs (Courtneidge 2003). Further analysis of each of these novel clones in mammalian cells confirmed that they were indeed Src substrates. One of these substrates, Tks5, is the subject of our current research and is described here.

\section{Tks5: A Sre SUBSTRATE AND SCAFFOLD PROTEIN}

The partial cDNAs we isolated from the Src substrate screen were designated Tks, for tyrosine kinase substrate. Conceptual translation of one such clone, Tks5, showed that it contained several possible Src phosphorylation sites and part of an SH3 domain (Lock et al. 1998). A fulllength clone of Tks5 was then isolated and sequenced.
Two distinct Tks5 transcripts were identified in a mouse embryonic cDNA library. Each transcript encodes a protein with an amino-terminal phox homology (PX) domain, five SH3 domains, and multiple proline-rich motifs. The larger transcript differs from the smaller by the presence of two alternative splices, positioned each side of SH3\#1 (Fig. 1). We have yet to explore fully the utilization of these transcripts, although our preliminary data suggest that fibroblasts in culture predominantly express a form of Tks5 containing only the second alternatively spliced exon. No catalytic domain was found in the protein, and it is therefore designated as a scaffolding or adapter protein, whose function is to interact with other proteins and lipids (see below).

We initially named the protein encoded by this locus Fish (for five SH3 domains). However, because of the potential for confusion, and the difficulty this name creates when searching databases, we have now reverted to the designation Tks5. Note also that in many databases, the gene encoding Tks5 has been given the name SH3MD1.

At the time that we originally cloned Tks5, which is located on human chromosome 10, we found no closely related sequences, although $\mathrm{p} 47^{\text {phox }}$, a cytoplasmic component of the NADPH oxidase system of neutrophils, did show some similarity in the arrangement of its PX domain and two SH3 domains. However, in 2004, a sequence (derived from a mouse dendritic cell cDNA library) that more closely resembled Tks5, with an amino-terminal PX domain followed by three SH3 domains, was deposited in databases. Some months ago, genomic databases were used to map this gene to human chromosome 5 , and to update and extend it, such that it is now predicted to contain four SH3 domains. Although this gene product remains at this point hypothetical, the fact that similar sequences are conserved in genomes from fish to man suggests that it is a genuine gene. We propose to name the protein Tks4. It is of note that Tks4 shares none of the putative Src phosphorylation sites found in Tks5, although it does have a 

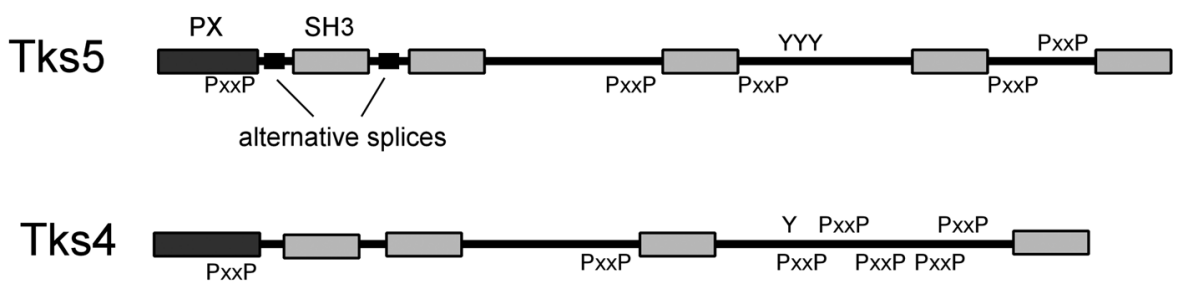

Figure 1. Domain map of Tks5 and the related protein Tks4. Black boxes represent phox homology (PX) domains, and gray boxes denote Src homology 3 (SH3) domains. Proline-rich motifs matching the consensus for SH3 domain binding are denoted PxxP, and each potential Src phosphorylation site is marked with a Y.

distinct canonical Src phosphorylation and $\mathrm{SH} 2$ domain binding site (YEEI) in the long linker sequence between the third and fourth SH3 domains. We are currently deriving a cDNA clone of Tks4 in order to study it further.

We observed that the Tks5 mRNA was generally expressed, although it was low in spleen and absent in testis (Lock et al. 1998). We have subsequently found, however, that some cell lines in culture fail to express the Tks5 protein, even when they contain the mRNA. Still others lack mRNA expression (Seals et al. 2005). This suggests that Tks5 expression is likely to be regulated at several levels, both transcriptionally and posttranscriptionally. We are currently exploring this in more depth. Tks5 is phosphorylated by Src in Src-transformed fibroblasts, and in normal cells in response to various stimuli, including growth factors and disruptors of the actin cytoskeleton (Abram et al. 2003). Cytoskeletal disruption in fibroblasts lacking Src, Fyn, and Yes did not result in Tks5 phosphorylation (C. Abram and S.A. Courtneidge, unpubl.). Thus, to date we have no evidence that Tks5 is a substrate of tyrosine kinases other than those of the Src family.

\section{Tks5 LOCALIZES TO PODOSOMES IN NORMAL AND CANCER CELLS}

In mouse fibroblasts, Tks5 has a predominantly cytoplasmic localization. However, we noticed a striking relocalization of Tks5 to the ventral membrane when these fibroblasts were transformed by Src (Fig. 2). Tks5 colocalized with F-actin in structures known as rosettes of po-
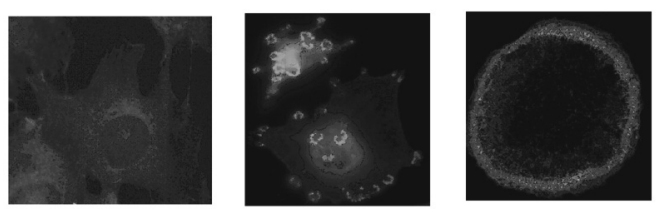

Tks5
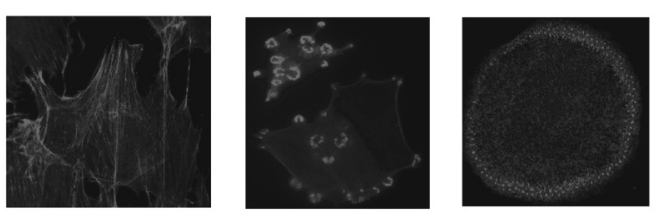

F-actin

NIH-3T3 dosomes (Abram et al. 2003). Podosomes, also known as invadopodia, have been implicated in both tumor cell invasion and the control of proteolysis (David-Pfeuty and Singer 1980; Tarone et al. 1985; Chen 1989; Linder and Aepfelbacher 2003). They are fine ventral protrusions of the plasma membrane, with an actin core, and rich in actin regulatory proteins. Indeed, several studies have shown that the formation of podosomes requires actin turnover. Podosomes also contain several proteases and have been implicated in degradation of the extracellular matrix (ECM). Podosomes are found in many cancer cells, including Src-transformed fibroblasts, as well as in invasive human carcinomas, including breast cancers and melanomas (Kelly et al. 1994; Chen 1996).

Podosomes have also been described in many normal cell types whose physiological roles require motility and ECM degradation, including osteoclasts, macrophages, endothelial cells, and vascular smooth muscle cells (Linder and Aepfelbacher 2003; Buccione et al. 2004). We have yet to examine each of these cell types for Tks 5 expression and localization, but, together with Drs. Elson and Geiger (Weizmann Institute), we have detected Tsk5 in the podosomes of mature osteoclasts (Fig. 2). Our current studies have focused on a role for Tks5 in the invasive nature of cancer cells, but it will be interesting in the future to dissect its role in cells such as osteoclasts.

\section{Tks5 IS NEEDED FOR PODOSOME FORMATION AND INVASION IN CANCER CELLS}

Malignant tumors are distinguished from their benign counterparts by their ability to metastasize. The process of metastasis involves many steps, including escape from the tissue in which the tumor first arises, passage into the vasculature, exit from the vasculature and movement into a distant tissue, followed by growth at this site. There are two properties in particular that propel this metastatic phenotype: motility and the promotion of proteolysis (Ridley 2000; Chang and Werb 2001). Proteases are not just involved in the degradation of the specialized ECM called basement membrane that surrounds organs, but are also responsible for the turnover and remodeling of ECM that occurs during tumor growth. Several proteases have been implicated in the process of metastasis, including both metalloproteases (particularly of the matrix type) and serine proteases (particularly urokinase plasminogen activator; uPA) (Blasi 1997; McCawley and Matrisian

Figure 2. The subcellular localization of Tks5. Cultured NIH$3 \mathrm{~T} 3$ cells $(l e f t), \mathrm{T} 3$ cells transformed with an activated form of Src (middle), and mature osteoclasts (right), were fixed and stained for Tks5 (top) or actin (using phalloidin, bottom). 
2000). Although tumor cells often themselves secrete the required proteases, it is also clear that tumor cells can orchestrate the production and activation of proteases by host cells in the tumor microenvironment, such as macrophages and osteoclasts (van Kempen et al. 2003). Intriguingly, each of these cell types can form podosomes (Linder and Aepfelbacher 2003).

We tested whether Tks 5 was required for the formation of podosomes, and for the invasive phenotype (Seals et al. 2005). We generated clones of Src-transformed NIH-3T3 cells expressing a short hairpin RNA vector targeting Tks5, which on average showed a fourfold reduction in Tks5 expression. Cell growth was not affected in the knockdown cells. We fixed and stained these cells with fluorescent phalloidin, to visualize F-actin. Whereas the parental cells and control clones (transfected with an empty vector) contained many rosettes of podosomes, these structures were only rarely observed in the knockdown cells. Rather, the cells appeared to regain some actin stress fibers and focal adhesions. Furthermore, the knockdown cells failed to elicit robust ECM degradation and were poorly invasive through Matrigel, although chemotaxis was unaffected (Fig. 3 and data not shown). Taken together, these data suggest that Tks5 is required both for podosome formation and for the invasive properties of the cells.

We next addressed whether Tks5 was expressed in human cancer cell lines and tissues (Seals et al. 2005). We found measurable expression of Tks5 in three invasive breast cancer cells. In contrast, only barely detectable levels were evident in two breast cancer cell lines that are considered poorly invasive. A similar correlation between Tks5 expression and invasiveness was seen in three prostate cancer cell lines (D. Seals et al., unpubl.). All melanoma cell lines tested expressed Tks5. Furthermore, we detected Tks5 expression in several samples of paraffin-embedded cancers, including breast, prostate, and melanoma (in collaboration with Dr. James Resau,
Van Andel Research Institute). We used short interfering RNAs to probe a possible role for Tks5 in the invasive ability of human cancer cells, particularly breast cancers and melanomas. In each case, we found that inhibition of Tks5 expression reduced the invasiveness of the cells through Matrigel, to the same extent as protease inhibitors. Although the sample sizes were small in these analyses, they do suggest that further study of the relationship between Tks5 expression, invasiveness, and cancer type and stage is warranted.

In other experiments, we have shown that the introduction of Tks5 into cells that do not normally express it (for example, T47D breast cancer cells), along with activated Src, results in the formation of podosomes (Seals et al. 2005). Together, our data suggest that Tks5 is critical for the formation of podosomes. Furthermore, our experiments provide further support for the conclusion that proteolytic degradation of the ECM, and invasive behavior, require podosomes.

\section{THE SCAFFOLDING FUNCTIONS OF TKS5}

Given that Tks5 lacks catalytic activity, it must derive its ability to drive the formation of podosomes from its interaction with other molecules. We have begun to characterize these molecules. We first analyzed the PX domain (Abram et al. 2003). Since other PX domains bind to phosphorylated inositol lipids (Sato et al. 2001; Wishart et al. 2001; Xu et al. 2001; Ellson et al. 2002), we tested the ability of the PX domain of Tks 5 to bind lipids. We found that, in vitro, the PX domain shows strong binding to both $\mathrm{PI} 3 \mathrm{P}$ and $\mathrm{PI} 3,4 \mathrm{P}_{2}$. When expressed in normal fibroblasts, the isolated PX domain of Tks5 shows a punctate intracellular distribution, consistent with the predominantly endosomal localization of PI3P. In Src-transformed cells, however, the PX domain is targeted to podosomes, suggesting that perhaps in this case it is associating with $\mathrm{PI} 3,4 \mathrm{P}_{2}$. Although these data might
A
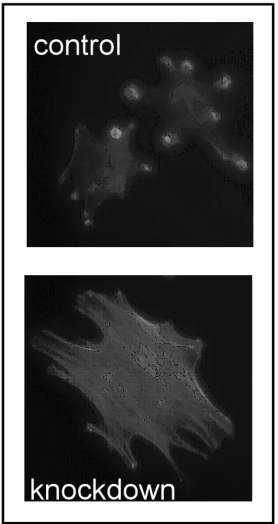

B

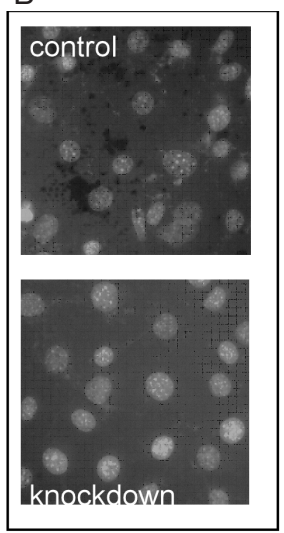

C

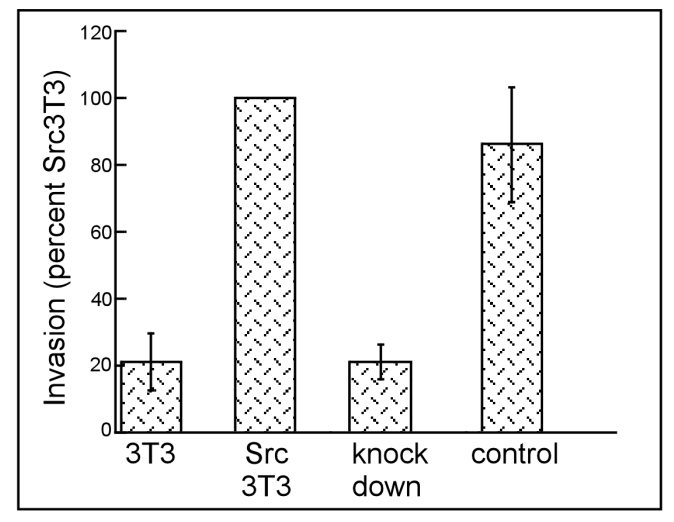

Figure 3. Tks5 is required for podosome formation, ECM degradation, and invasion. (A) Src-3T3 cells stably transfected with control vector (control), or a vector containing shRNA targeting Tks5 (knockdown), were fixed and stained with phalloidin to visualize the actin cytoskeleton. $(B)$ Control and knockdown cells were plated onto coverslips coated with FITC-gelatin. After 24 hr, degradation of the gelatin was evaluated by fluorescence microscopy. Cells were also stained with DAPI to visualize the nuclei. $(C)$ Parental 3T3 and Src-3T3 cells, as well as control and knockdown cells, were assayed for their ability to invade through Matrigel, using a Boyden chamber assay. 
suggest that Tks5 is targeted to podosomes via a PX domain:lipid interaction, we note that its PX domain also contains a polyproline motif that might allow it to associate with $\mathrm{SH} 3$ domain-containing proteins. We are currently generating the appropriate mutants that will allow us to test the relative roles of lipid and protein binding in targeting Tks5 to podosomes.

We have also begun to characterize the proteins that bind to the SH3 domains of Tks5. The first technique we used was phage display, using phage carrying inserts from a mammalian cDNA library (Abram et al. 2003). The top hit in this analysis was a carboxy-terminal fragment of ADAM19, which is a member of the ADAMs family of metalloproteases. Subsequent biochemical analyses in mammalian cells confirmed this association and also revealed an association between Tks5 and ADAMs 12 and 15. The ADAMs family is a group of transmembrane proteins, already known to associate with several signaling molecules via their proline-rich tails, and with functions as diverse as shedding of growth factors and cytokines, motility, and cell fate determination (Seals and Courtneidge 2003). We determined the effect of Tks5 association on ADAM12 localization. Whereas ADAM12 showed a predominantly intracellular localization in normal cells (presumably because of its association with the endoplasmic reticulum), in a breast cancer cell line (and in Src-tranformed fibroblasts), some ADAM12 was localized to podosomes (Fig. 4). This relocalization has the effect of orienting the protease domain and the integrin binding domains of ADAM12 to face the extracellular milieu. It will be of great interest in the future to determine whether ADAM12, or the other ADAMs that associate with Tks5, play any role in podosome formation and/or function, and in cancer cell invasion.

We have just begun to isolate binding partners for the other SH3 domains of Tks5. One method we are currently using entails incubating cell extracts with each SH3 domain expressed as a GST fusion protein, resolving the associated proteins by gel electrophoresis, and sequencing
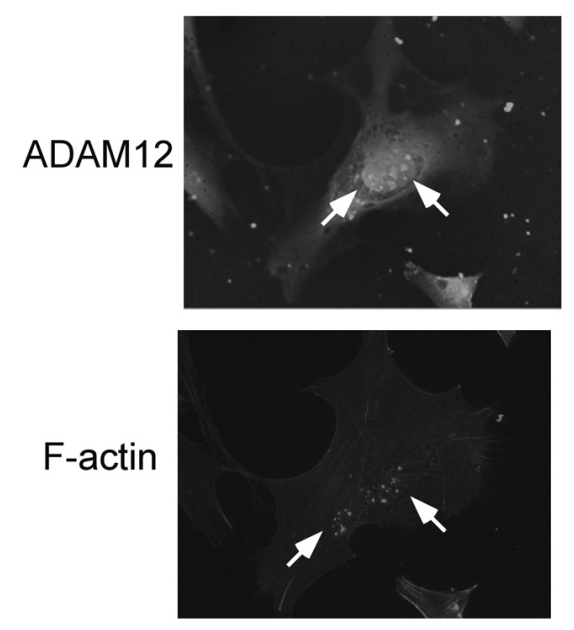

Figure 4. ADAM12 is localized to podosomes in breast cancer cells. Hs578T breast cancer cells were stained with antibodies against ADAM12, or with phalloidin. The arrows indicate the positions of clusters of podosomes. the proteins by mass spectrometry (in collaboration with Greg Cavey at the Van Andel Research Institute). We are also using co-immunoprecipitation analyses to probe the association of Tks5 with proteins known to be present in podosomes. Although our data are preliminary, and as yet unpublished, they are intriguing. For example, we have detected associations with dynamin, N-WASp, and focal adhesion kinase, all proteins shown by other investigators to be required for podosome formation.

\section{CONCLUSIONS}

In the last few years, we have begun the characterization of an intriguing scaffold protein and Src substrate, Tks5. In normal fibroblasts, Tks5 is predominantly cytoplasmic. We have found that it becomes tyrosine phosphorylated by Src family kinases following mitogen stimulation, and upon disruption of the actin cytoskeleton. However, the consequences of this phosphorylation, as well as any potential role for Tks5 in the response to mitogens, have yet to be explored.

In Src-transformed cells, Tks5 is constitutively phosphorylated on tyrosine residues, and associated with podosomes. We know that the PX domain of Tks5 is both necessary and sufficient for podosome localization. Yet in normal cells, in the context of the full-length protein, the PX domain is not available to bind lipid. This is possibly due to intramolecular interactions between the PX domain and one of the SH3 domains, as has been reported for $\mathrm{p} 47^{\text {phox }}$ (Hiroaki et al. 2001). Our current working hypothesis is that upon Src transformation, this intramolecular association is weakened, freeing the PX domain for intermolecular interactions. We are currently testing the possible role of the tyrosine phosphorylation of Tks 5 in this process.

Our studies show that in vitro, Tks5 is required for invasive behavior and for podosome formation. What about in vivo? We have preliminary evidence that reduction in Tks5 expression affects the subcutaneous growth of Srctransformed fibroblasts in immunocompromised mice (D. Seals and S.A. Courtneidge, unpubl.). We are currently generating a Tks5-GFP fusion protein, so that we may visualize its localization, and the morphology of podosomes, in 3-dimensional culture systems and in tumor explants. However, a full understanding of Tks 5 function in cancer progression will require appropriate animal models. To this end, we are in the process of creating mice expressing a conditional allele of Tks5 so that we may ablate Tks5 expression in either tumor cells or host cells such as macrophages or endothelial cells, and test the effect in several mouse models of tumorigenesis. Such mice will also facilitate our analysis of Tks5 during embryogenesis.

\section{ACKNOWLEDGMENTS}

We thank former and current lab members Clare Abram, Laila Al-Duwaisan, Rebecca Gordon, Peter Lock, Lisa Maurer, Therese Roth, and Dan Salinsky; and our collaborators Greg Cavey, Ari Elson, Benjamin Geiger, and James Resau, for their contributions to this research. 


\section{REFERENCES}

Abram C.L., Seals D.F., Pass I., Salinsky D., Maurer L., Roth T.M., and Courtneidge S.A. 2003. The adaptor protein Fish associates with members of the ADAMs family and localizes to podosomes of Src-transformed cells. J. Biol. Chem. 278: 16844.

Blasi F. 1997. uPA, uPAR, PAI-1: Key intersection of proteolytic, adhesive and chemotactic highways? Immunol. Today 18: 415 .

Buccione R., Orth J.D., and McNiven M.A. 2004. Foot and mouth: Podosomes, invadopodia and circular dorsal ruffles. Nat. Rev. Mol. Cell Biol. 5: 647.

Chang C. and Werb Z. 2001. The many faces of metalloproteases: Cell growth, invasion, angiogenesis and metastasis. Trends Cell Biol. 11: S37.

Chen W.T. 1989. Proteolytic activity of specialized surface protrusions formed at rosette contact sites of transformed cells. $J$. Exp. Zool. 251: 167.

1996. Proteases associated with invadopodia, and their role in degradation of extracellular matrix. Enzyme Protein 49: 59.

Courtneidge S.A. 2003. Isolation of novel Src substrates. Biochem. Soc. Trans. 31: 25.

David-Pfeuty T. and Singer S.J. 1980. Altered distributions of the cytoskeletal proteins vinculin and alpha-actinin in cultured fibroblasts transformed by Rous sarcoma virus. Proc. Natl. Acad. Sci. 77: 6687.

Ellson C.D., Andrews S., Stephens L.R., and Hawkins P.T. 2002. The PX domain: A new phosphoinositide-binding module. J. Cell Sci. 115: 1099.

Frame M.C. 2002. Src in cancer: Deregulation and consequences for cell behaviour. Biochim. Biophys. Acta 1602: 114.

Hiroaki H., Ago T., Ito T., Sumimoto H., and Kohda D. 2001. Solution structure of the PX domain, a target of the SH3 domain. Nat. Struct. Biol. 8: 526.

Kelly T., Mueller S.C., Yeh Y., and Chen W.T. 1994. Invadopodia promote proteolysis of a wide variety of extracellular ma- trix proteins. J. Cell. Physiol. 158: 299.

Linder S. and Aepfelbacher M. 2003. Podosomes: Adhesion hotspots of invasive cells. Trends Cell Biol. 13: 376.

Lock P., Abram C.L., Gibson T., and Courtneidge S.A. 1998. A new method for isolating tyrosine kinase substrates used to identify Fish, an SH3 and PX domain-containing protein, and Src substrate. EMBO J. 17: 4346.

Martin G.S. 2001. The hunting of the Src. Nat. Rev. Mol. Cell Biol. 2: 467.

McCawley L.J. and Matrisian L.M. 2000. Matrix metalloproteinases: Multifunctional contributors to tumor progression. Mol. Med. Today 6: 149.

Ridley A. 2000. Molecular switches in metastasis. Nature 406: 466.

Sato T.K., Overduin M., and Emr S.D. 2001. Location, location, location: Membrane targeting directed by PX domains. Science 294: 1881.

Seals D.F. and Courtneidge S.A. 2003. The ADAMs family of metalloproteases: Multidomain proteins with multiple functions. Genes Dev. 17: 7.

Seals D.F., Azucena, Jr. E.F., Pass I., Tesfay L., Gordon R., Woodrow M., Resau J.H., and Courtneidge S.A. 2005. The adaptor protein Tks5/Fish is required for podosome formation and function, and for the protease-driven invasion of cancer cells. Cancer Cell 7: 155.

Tarone G., Cirillo D., Giancotti F.G., Comoglio P.M., and Marchisio P.C. 1985. Rous sarcoma virus-transformed fibroblasts adhere primarily at discrete protrusions of the ventral membrane called podosomes. Exp. Cell Res. 159: 141.

van Kempen L.C., Ruiter D.J., van Muijen G.N., and Coussens L.M. 2003. The tumor microenvironment: A critical determinant of neoplastic evolution. Eur. J. Cell Biol. 82: 539.

Wishart M.J., Taylor G.S., and Dixon J.E. 2001. Phoxy lipids: Revealing PX domains as phosphoinositide binding modules. Cell 105: 817.

Xu Y., Seet L.F., Hanson B., and Hong W. 2001. The Phox homology (PX) domain, a new player in phosphoinositide signalling. Biochem. J. 360: 513. 


\section{$\$_{\text {SSH\& }}^{\infty} \mathrm{CS}$ Cold Spring Harbor Symposia SYMPOSIA on Quantitative Biology}

\section{The Src Substrate Tks5, Podosomes (Invadopodia), and Cancer Cell Invasion}

S.A. COURTNEIDGE, E.F. AZUCENA, JR., I. PASS, et al.

Cold Spring Harb Symp Quant Biol 2005 70: 167-171

Access the most recent version at doi:10.1101/sqb.2005.70.014

References This article cites 24 articles, 6 of which can be accessed free at:

http://symposium.cshlp.org/content/70/167.full.html\#ref-list-1

License

Email Alerting Receive free email alerts when new articles cite this article - sign up in

Service the box at the top right corner of the article or click here. 